\title{
Hardness Enhancement and Corrosion Current of Multilayer Coatings Based on Titanium Nitride
}

\author{
Arturo Talledo ${ }^{1 *}$, Junior Asencios ${ }^{1}$, Karin Paucar ${ }^{1}$, Alcides López ${ }^{1}$, Carsten Benndorf ${ }^{1}$, Rolando Nuñez ${ }^{2}$ and Jan \\ Petersen $^{3}$ \\ 1. Facultad de Ciencias, Universidad Nacional de Ingeniería, Av. Tupac Amaru 210, Lima25, Perú \\ 2. Departamento Ingeniería Mecánica, Pontificia Universidad Católica del Perú, Av Universitaria s/n Lima 32, Perú \\ 3. Fraunhofer IST, BienroderWeg 54 E, 38108 Braunschweig, Germany
}

\begin{abstract}
In this article we explore the possibilities to obtain hardness enhancement in three different types of multilayer system: TiN/AlN, TiN/NbN and TiN/TaN, produced by bias-dc magnetron sputtering under the same deposition conditions. Multilayer structure and chemical composition were confirmed by GDOES (Glow discharge optical emission spectroscopy). Crystalline structure of component layers was studied by XRD (X-ray diffraction). Microhardness was measured by using a classic Vickers microdurometer and SEM (Scanning electron microscopy), as well as, by nanoindentation. Corrosion current was determined by Tafel extrapolation method.
\end{abstract}

Key words: Multilayers, hardness enhancement, negative bias, nanocrystalline structure, interfaces.

\section{Introduction}

Superhard and ultrahard materials are defined [1] as those with Vickers hardness in the ranges 40-80 GPa and more than $80 \mathrm{GPa}$ respectively. Since 1995, after original finding of Veprek and Reiprich $[2,3]$ about the hardness of nanocomposite: nc-TiN-a- $\mathrm{Si}_{3} \mathrm{~N}_{4}$, an intense research on these materials has started. These materials have been produced as nanostructured coatings on high speed steel, silicon and other substrates.

Several structures have been developed for superhard and ultrahard nanostructured coatings such as: (1) nanocomposites coatings [4, 5], made for instance by co-sputtering of two or more targets in an atmosphere of argon and nitrogen; (2) nanoscale multilayers [6-8], or superlattices, where layers of titanium nitride and other nitrides with wavelengths less than $10 \mathrm{~nm}$ are superposed one on the top of other; (3) graded metal nitrides [9], where the relative content of two metals changes gradually in the coating.

In This paper we describe the production by $\mathrm{dc}$

\footnotetext{
*Corresponding author: Arturo Talledo, Dr. of physics, research fields: tribology and thin films. E-mail: atalledo@uni.edu.pe.
}

magnetron sputtering of multilayers based on titanium nitride on HSS (High speed steel) substrates. Specifically we report on the production of TiN/AIN, $\mathrm{TiN} / \mathrm{NbN}$ and TiN/TaN multilayers. Chemical analysis of each layer was made by GDOES (Glow discharge optical emission spectroscopy). We describe also structural characterization by XRD (X-ray diffraction). Hardness was measured by using a Vickers microdurometer as well as by using nanoindentation. Electrochemical corrosion measurements are also reported.

\section{Experimental Procedures}

TiN/AlN, TiN/NbN and TiN/TaN multilayers were produced by dc magnetron sputtering technique. We used two sputter guns: one with a titanium target and the other one with other metal target, specifically, aluminum, niobium or tantalum. All targets were 3 inches diameter and $4 \mathrm{~mm}$ thick; their purity was better than $99.99 \%$. Substrates were high speed steel. The multilayers were produced by maintaining first the substrate above the titanium target for $5 \mathrm{~min}$, then rotating the substrate holder and maintaining it during 
1 min above the other-metal target; and repeating the process until we obtained twenty layers of TiN and 19 layers of the other nitride, i.e. the first and last layers are made of a titanium nitride. The process always started with sputtering a titanium adhesion layer during 2 min sputtering. During nitrides deposition, the sputter atmosphere was always $3.5 \times 10^{-4}$ mbar nitrogen and total pressure (argon + nitrogen) $5.6 \times 10^{-3}$ mbar. Current deposition for titanium nitride was always 600 $\mathrm{mA}$ and for the other nitrides $300 \mathrm{~mA}$. The substrates were always heated at $400{ }^{\circ} \mathrm{C}$ and biased at $-100 \mathrm{~V}$.

Thickness of each layer was first estimated by deposition time and previous calibration of deposition rate for each material. GDOES was used to analyze the chemical composition of each layer and verify the thickness of each layer. For that, Horiba Profiler 2 was used operating at $650 \mathrm{~Pa}$ and $35 \mathrm{~W}$ rf-power. The crystalline structure of our coatings was studied by XRD. A Phillips diffractometer Xpert 2000 with lambda $0.154 \mathrm{~nm}$ from $K_{\alpha}$ cooper transition was used in Bragg Bretano configuration. Coatings on steel were indented during $25 \mathrm{~s}$, by using a Buehler microdurometer. The indented samples were then taken to a SEM (Scanning electron microscopy) equipment in where diagonal of the indentations were measured. A FEI model Quanta 650 SEM equipment providing a calibrated microscale was used. A second measurement of hardness was made by nanoindentation with a Berkovich tip, obtaining simultaneously the elastic modulus. We used multiple partial unloading indents with a maximum load of $15 \mathrm{mN}$ to retrieve data at 25 depth values per indent with 16 indents per sample. Evaluation of the data was made according to the Oliver-Pharr [10] model. An Gamry-Reference $\quad 600 \quad$ computer-controlled potentiostat/galvanostat/ZRA connected to a three-electrode cell was used for the measurements of electrochemical corrosion. The working electrode was the test material with an immersed area of $1.33 \mathrm{~cm}^{2}$, platinum and silver/silver chloride $(\mathrm{Ag} / \mathrm{AgCl})$ electrodes were used as the counter and reference electrodes, respectively. The solution inside the reference electrode compartment was potassium chloride $(\mathrm{KCl})$ with concentration of 3.0 mole/liter. The test solution was a naturally aerated $0.10 \mathrm{M}$ $\mathrm{Na}_{2} \mathrm{SO}_{4}-0.01 \mathrm{M} \mathrm{NaCl}$ solution at room temperature $\left(26^{\circ} \mathrm{C}\right)$. Potentiodynamic polarization curves were obtained after immersion in $0.10 \mathrm{M} \mathrm{Na}_{2} \mathrm{SO}_{4}-0.01 \mathrm{M}$ $\mathrm{NaCl}$ solution for $1 \mathrm{~h}$ using a scan rate of $0.166 \mathrm{mV} / \mathrm{s}$, from $-250 \mathrm{mV}$ until $+250 \mathrm{mV}$ with respect to the OCP (Open-circuit potential).

\section{Results}

From deposition rate of nitrides deposited at sputter conditions mentioned in previous section, we estimated the thickness for each layer. These results are shown in Table 1.

In Fig. 1, we show the results of chemical analysis by GDOES. The multilayers structure of the three samples is very well defined. We can see 9 layers of aluminium nitride intercalated with 10 layers of TiN in Fig. 2a, 19 layers of NbN with 20 layers of TiN in Fig. 2b and 19 layers of TaN with 20 layers of TiN in Fig. 2c. There are, however, some details to explain. When the distance in the direction from the top surface of the multilayers to the substrate increases, the intensity of peaks of the metal elements decreases. This effect is known as the crater effect and it is due to the loss of resolution when depth increases [11, 12]; the continuous changes in the shape of the crater after each interface induces a higher mixing of the layers. So, quantitative information of the chemical composition and the width of layers only is reliable from the two first layers. We have obtained the thickness of a bilayer (wavelength) as the distance (in $\mathrm{nm}$ ) between the two first peaks corresponding to the metal ( $\mathrm{Al}, \mathrm{Nb}$ or $\mathrm{Ta}$ ) and the thickness of AlN, $\mathrm{NbN}$ or TaN layers as the width at half height of those peaks. We conclude that the chemical composition is very close to the stoichiometry of the corresponding nitrides expected and the thickness of the layers agree with those estimated in Table 1. 

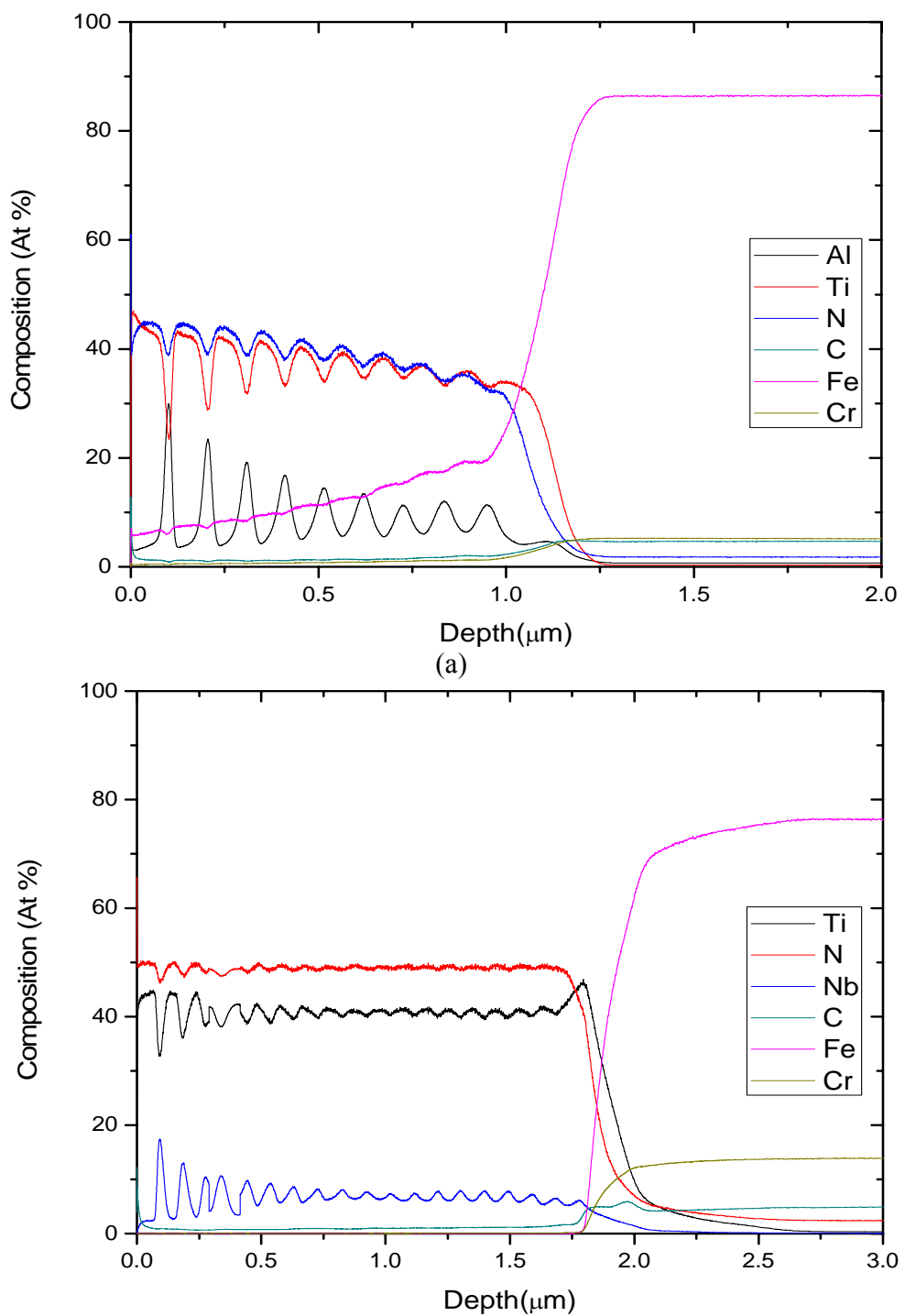

(b)

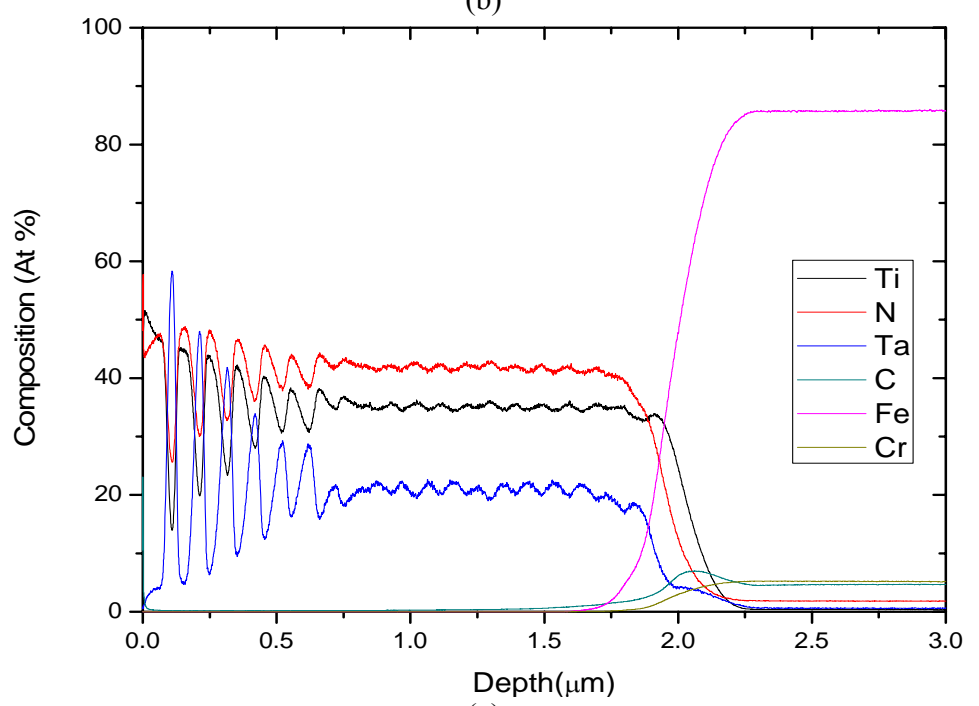

(c)

Fig. 1 GDOES analysis of multilayers systems (a) TiN/AIN, (b) TiN/NbN and (c) TiN/TaN on HSS-steel. 


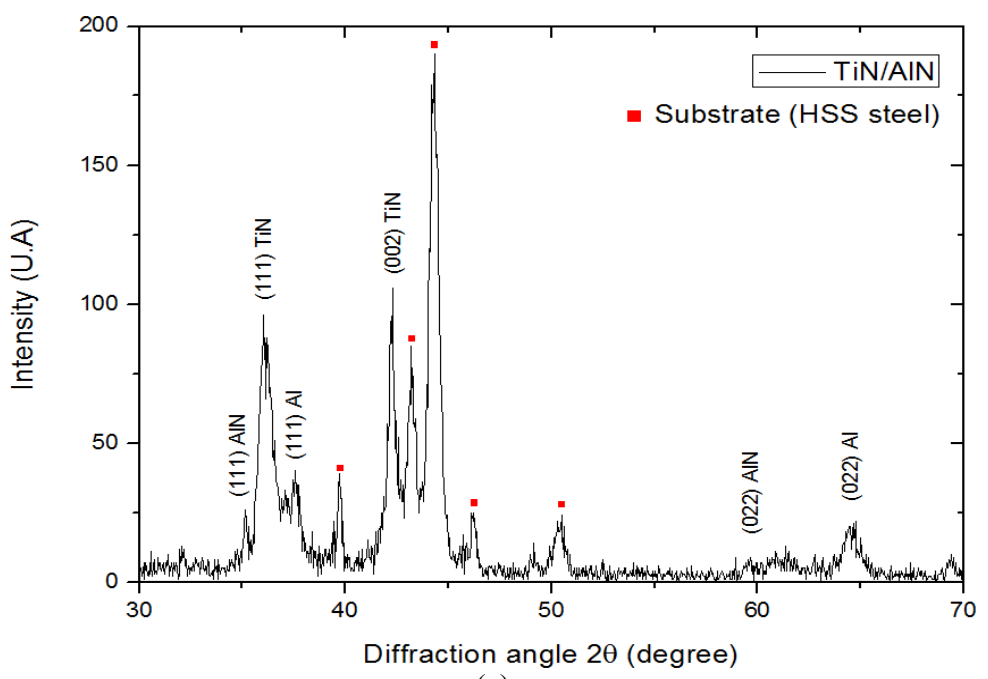

(a)

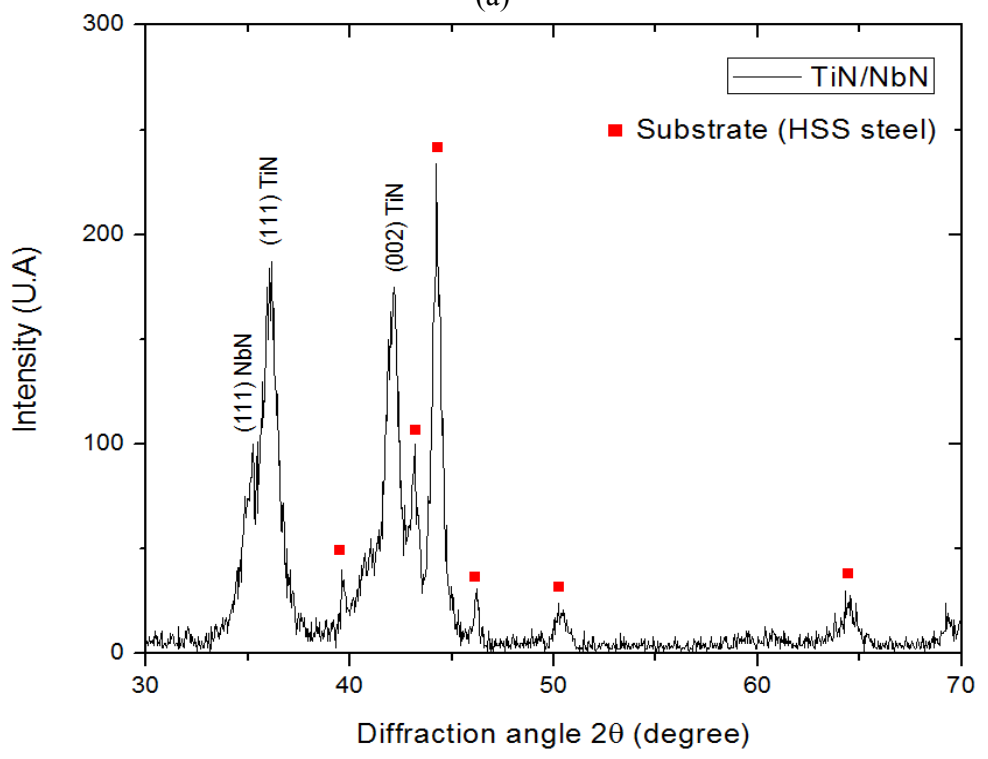

(b)

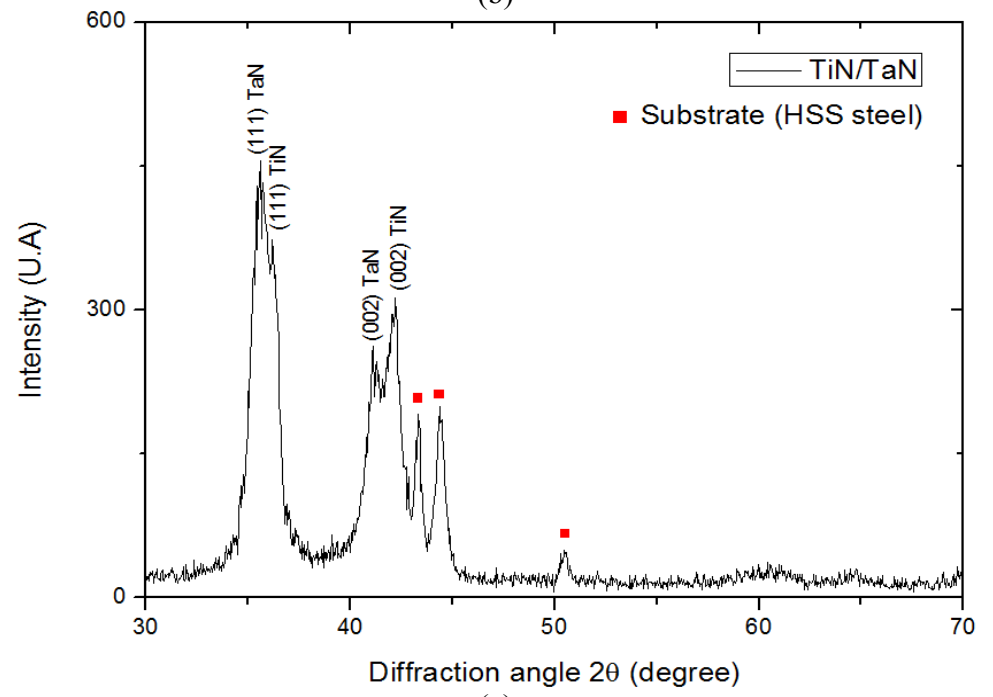

(c)

Fig. 2 X-ray diffractograms of multilayers (a) TiN/AIN, (b) TiN/NbN and (c) TiN/TaN on HSS steel. 
Table1 Estimation of each-layer thickness and total thickness of nitride coatings.

\begin{tabular}{lllll}
\hline & TiN thickness $(\mathrm{nm})$ & AlN, NbN and TaN thickness $(\mathrm{nm})$ & Number of bilayers & Total thickness $(\mu \mathrm{m})$ \\
\hline TiN/AlN & 80 & 13 & 10 & 1.0 \\
TiN/NbN & 80 & 13 & 20 & 1.9 \\
TiN/TaN & 80 & 16 & 20 & 1.9 \\
\hline
\end{tabular}

Fig. 2 shows XRD of a) 10 bilayers of TiN/AlN, b) 20 bilayers of TiN/NbN and c) 20 bilayers of TiN/TaN, all of them deposited on HSS-steel substrates. All the nitrides show nanocrystalline-rock-salt structure and the lattice parameters are: $0.430 \mathrm{~nm}$ for TiN, $0.441 \mathrm{~nm}$ for AlN, $0.478 \mathrm{~nm}$ for $\mathrm{NbN}$ and $0.437 \mathrm{~nm}$ for TaN. Grain size (D) for nanocrystalline multilayers can be estimated from broadening (B) of the diffraction lines and wavelength $(\lambda)$ of the $\mathrm{X}$-ray beam by the very well known Sherrer equation [13]:

$$
D=\frac{0.9 \lambda}{B \cdot \cos \theta}
$$

For TiN/AlN grain size of TiN is $11.0 \mathrm{~nm}$ and AlN is $9.8 \mathrm{~nm}$; For TiN/NbN grain size of TiN is $11.6 \mathrm{~nm}$ and $\mathrm{NbN}$ is $9.9 \mathrm{~nm}$; For TiN/TaN grain size of TiN is $12.1 \mathrm{~nm}$ and TaN is $8.2 \mathrm{~nm}$.

Fig. 3 shows SEM micrographies of Vickers indentations with loads $\mathrm{L}=50,10$ and $5 \mathrm{gf}$ for following samples: a) HSS-steel substrate, b) 10 TiN/AlN bilayers, c) $20 \mathrm{TiN} / \mathrm{NbN}$ bilayers and d) 20 TiN/TaN bilayers. From the size of the diagonals shown in the micrographies we have calculated the Vickers hardness which is shown in Table 2.

First, let us observe in Fig. 4 that the Vickers hardness of the HSS substrate is independent of the load in the range 100-10 gf. The value for $5 \mathrm{gf}$ increases in $20 \%$. This is a very well known indentation size effect [14] explained for the high dislocation density under the indentation tip. For large penetration depths the dislocations are distributed in larger volumes, so the dislocation density is smaller.

For the coated samples, we can observe that hardness increases noticeably for loads lesser than $50 \mathrm{gf}$ and they reach high values for $\mathrm{L}=5 \mathrm{gf}$. This can be explained for the very well known rule: A most real approximation to the hardness of the coating is obtained when ratio (indentation depth/coating thickness) $<0.1$.

Considering that the values of Vickers Hardness for titanium nitride and other nitrides, components of our coatings, are in the range $1,500-2,200 \mathrm{kgf} / \mathrm{mm}^{2}$, it is evident from our data in Fig. 5 and Table 2 that our multilayer coatings do show hardness enhancement compared to monolayer nitride coatings.

\section{Nanoindentation}

Hardness of our coatings was also measured by nanoindentation. The advantage of this method is essentially a smaller tip radius which allows for a more accurate measurement at small loads and low indentation depths to avoid the influence of the substrate hardness on the results. The values given in Table 3 are an average over 16 multiple-partial-unloading indents taken at an indentation depth region between 40 and $110 \mathrm{~nm}$. This region enable us to get an average over several layers of the multilayered samples without interference of the substrate. At lower depths there is a large scattering of data due to the predominance of surface roughness as well as indentation tip rounding effects. The parameter $\mathrm{H}^{3} / \mathrm{E}^{* 2}$ related to toughness of coatings, is also shown.

In Fig. 4, we show graphically the hardness values obtained from data in Table 2 vs load. Data for $100 \mathrm{gf}$ is also included. Table 4 shows the indentation depth in nanometers.

In Fig. 5 we show the results of multiple-partial-unloading indentation for the indents of maximum and minimum hardness.

The data show that the nanoindentation results for hardness (first row in Table 3) are consistently lower than the microhardness values measured with load $\mathrm{L}=$ $5 \mathrm{gf}$ (last column in Table 2). It is very well known that 

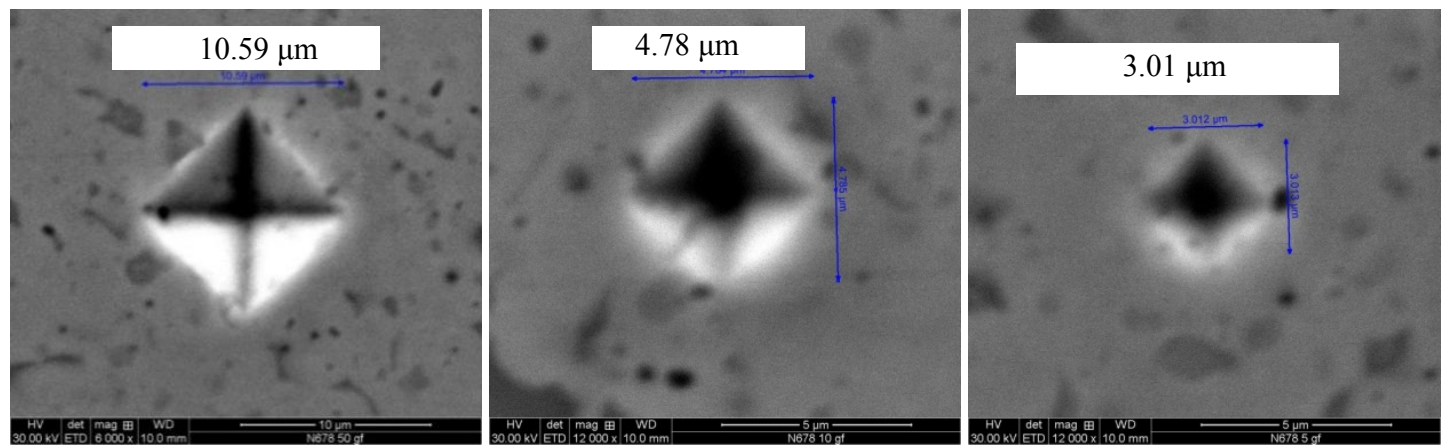

(a)
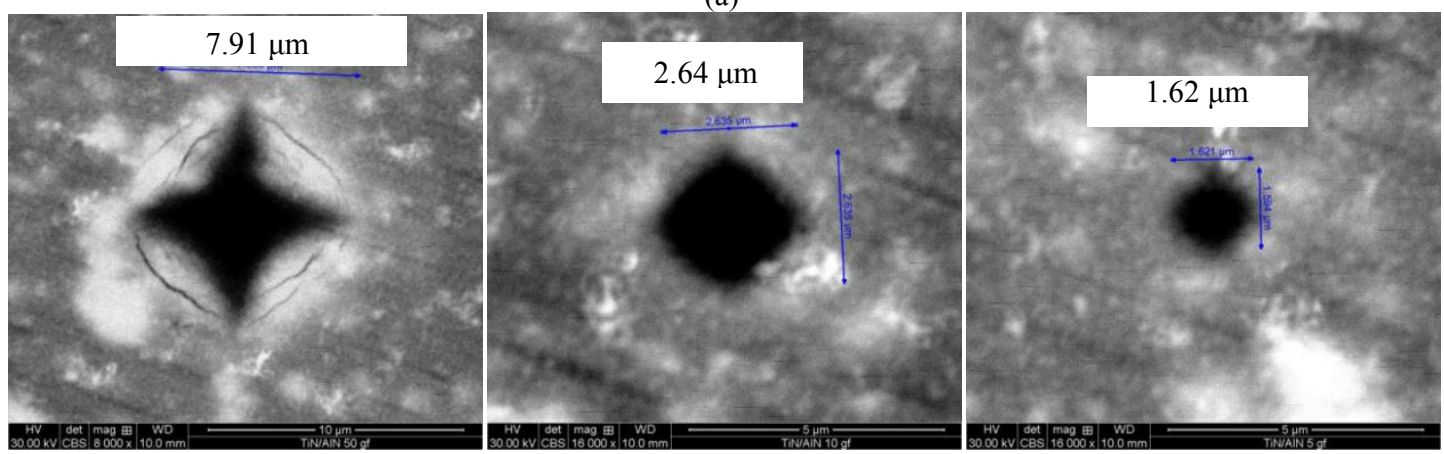

(b)
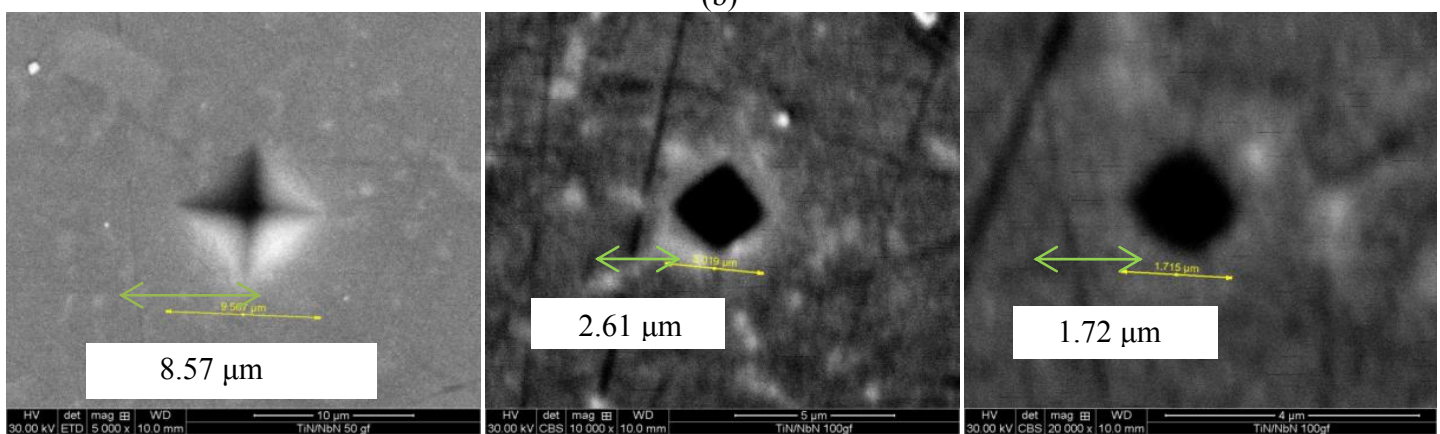

(c)
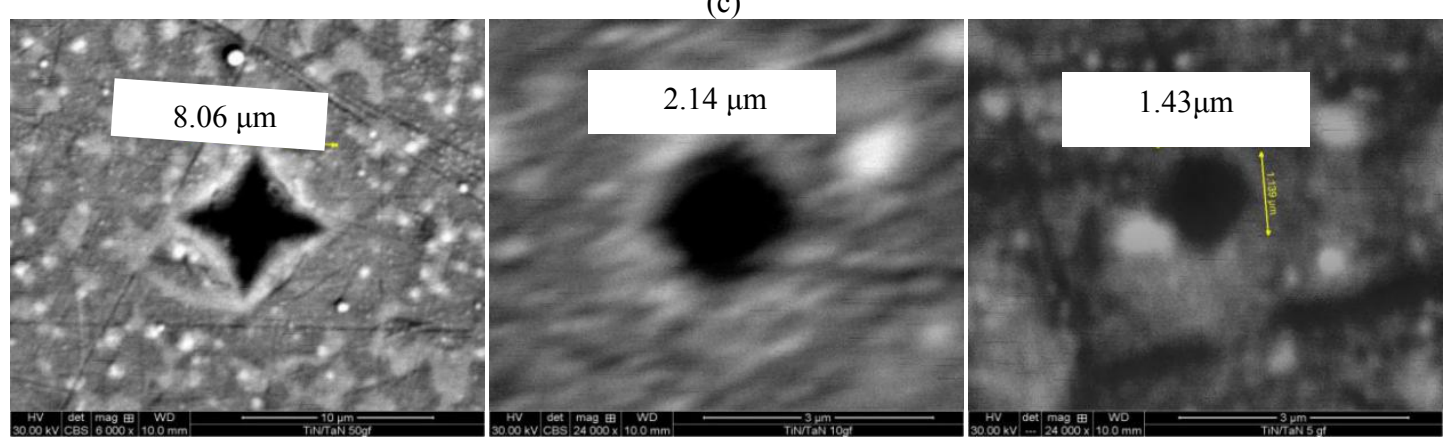

(d)

Fig. 3 SEM micrographies of 50, 10 and 5 gf -indentations on: (a) HSS substrate and multilayers, (b) TiN/AIN, (c) TiN/NbN and (d) TiN/TaN.

Table 2 Vickers hardness $\left(\mathbf{k g f} / \mathrm{mm}^{2}\right)$.

\begin{tabular}{llll}
\hline & 50 gf & 10 gf & 5 gf \\
\hline Substrate & 827 & 810 & 1022 \\
TiN/AlN (10 layers) & 1482 & 2670 & 3527 \\
TiN/NbN (20 layers) & 1262 & 2720 & 3151 \\
TiN/TaN (20 layers) & 1427 & 4017 & 4498 \\
\hline
\end{tabular}


Table 3 Average hardness.

\begin{tabular}{llll}
\hline & TiN/AlN & TiN/NbN & TiN/TaN \\
\hline $\mathrm{H}(\mathrm{GPa})$ & $29.4 \pm 3.4$ & $29.7 \pm 2.6$ & $31.3 \pm 1.8$ \\
$\mathrm{E}^{*}(\mathrm{GPa})$ & $618 \pm 94$ & $635 \pm 71$ & $553 \pm 40$ \\
& $0.07 \pm 0.03$ & $0.06 \pm 0.02$ & $0.01 \pm 0.02$ \\
\hline
\end{tabular}

Table 4 Indentation depth (nm).

\begin{tabular}{llll}
\hline & 50 gf & 10 gf & 5 gf \\
\hline Substrate & 1513 & 682 & 430 \\
TiN/AlN (10 layers) & 1128 & 377 & 231 \\
TiN/NbN (20 layers & 1367 & 291 & 246 \\
TiN/TaN (20 layers) & 1151 & 291 & 169 \\
\hline
\end{tabular}

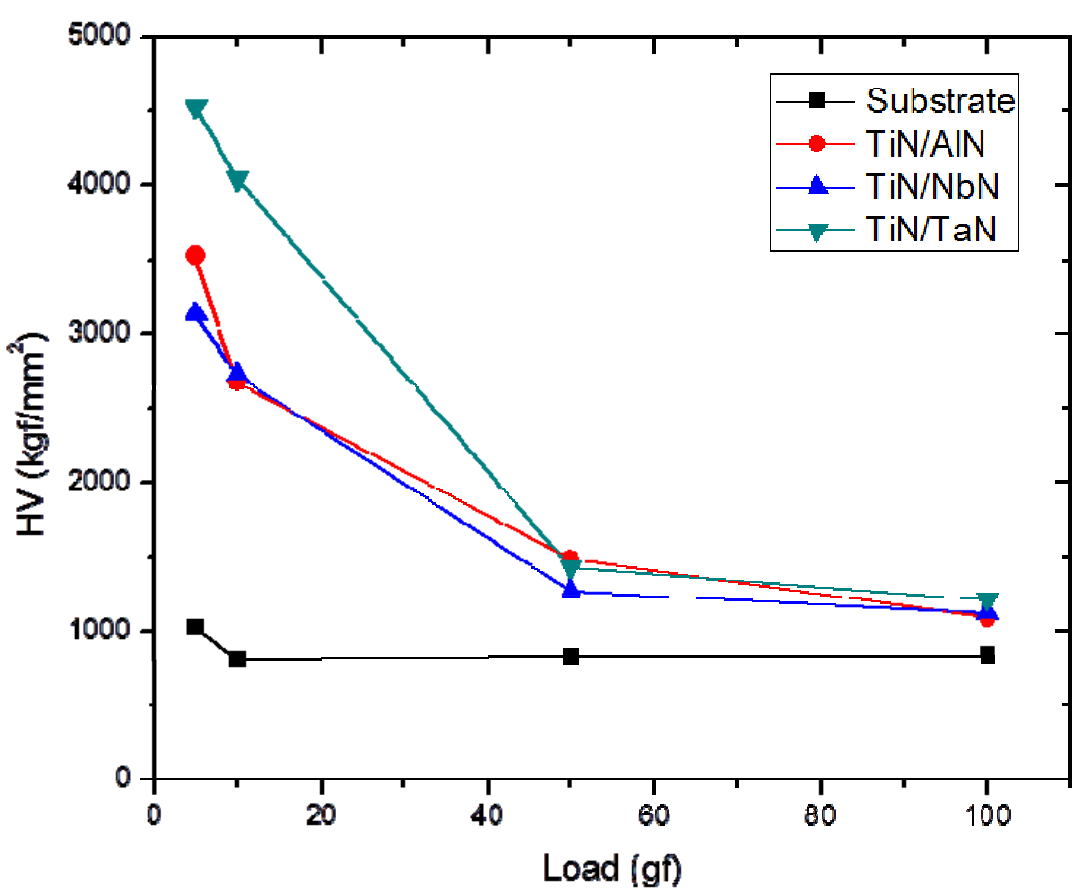

Fig. 4 Vickers Hardness vs load for HSS steel coated with multilayers according to inset.

there are discrepancies in hardness-measurement methods mainly due to the differences in tip geometry. Also the difference in indentation depth can have a large impact on the results. Generally, the larger indentation depth of the microhardness measurement could result in the interference of film hardness and substrate hardness, but this would lead to a lower hardness value due to the softer substrate. Also the microstructure, as well as, the intrinsic stress of the individual layers could vary during film growth. In any case, it is evident that all of our multilayer coatings show hardness enhancement. Considering the error margine, there is no significant difference in the hardness of the three different systems.

The scattering of the hardness values is quite large (see Fig. 5). This can also be seen in data by other authors. Following the discussion by N. Pattel et al. [15], one explanation can be the polycrystalline nature of our coatings. The hardness is greater in the $<111>$-direction than in $<100>$. Tipically, for hardness measurements a large scattering of data is observed for samples with a high surface roughness. Since there is a difference in contact area when the tip penetrates the surface on a peak or on a valley, there will be a difference result in the hardness. Depending on the roughness this effect can be significantly larger than 


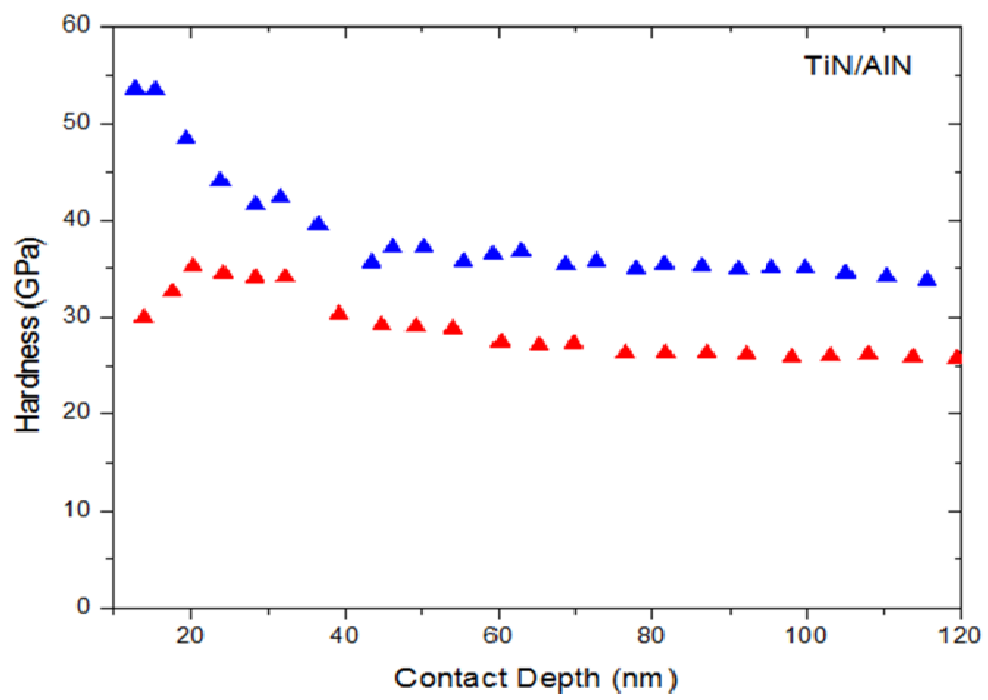

(a)

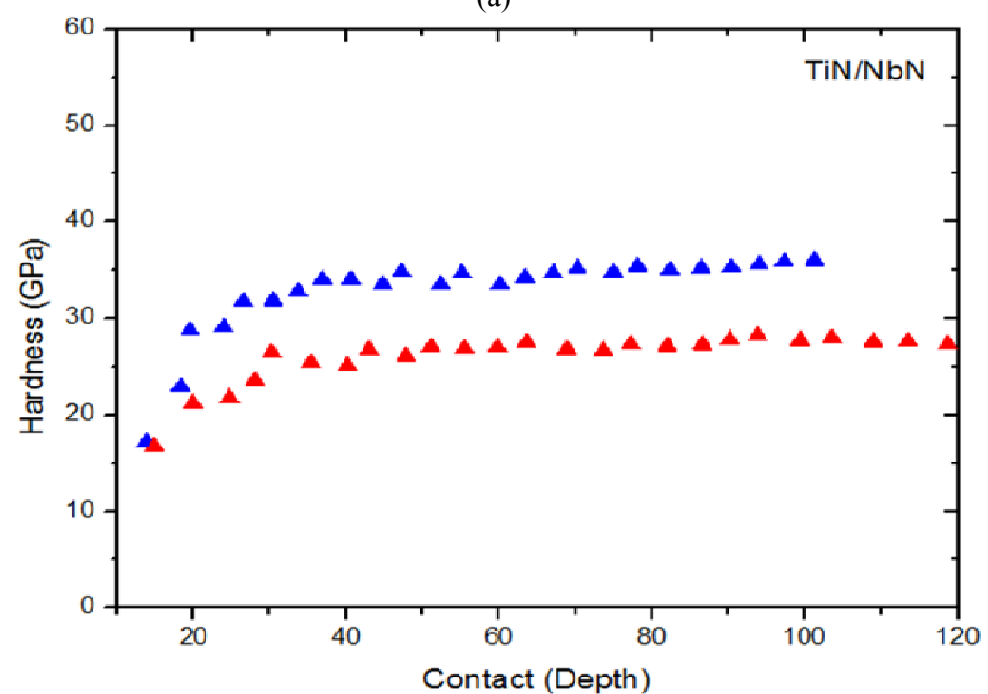

(b)

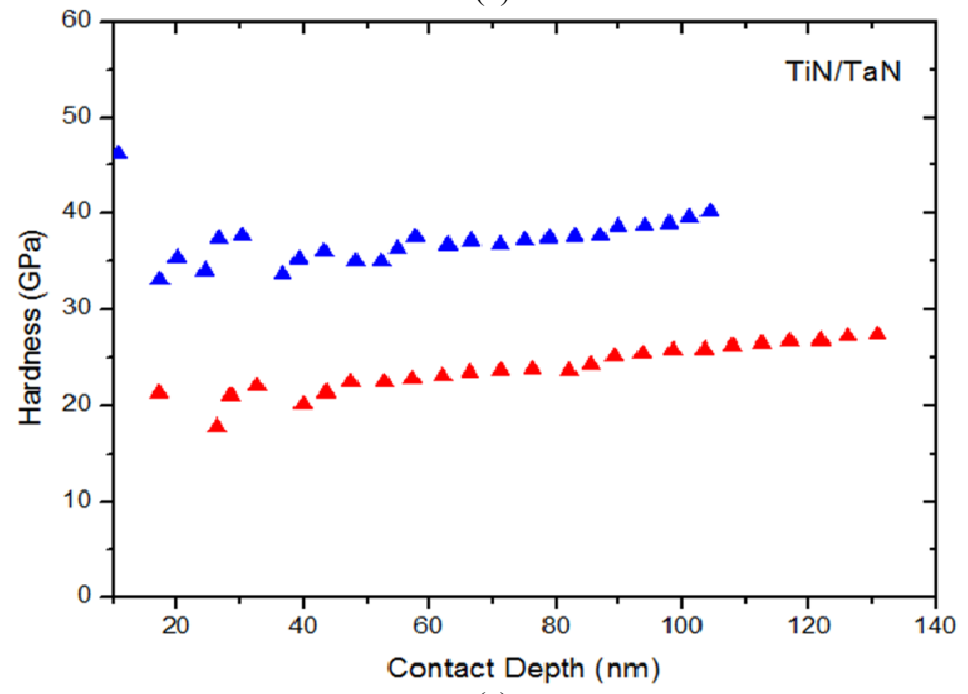

(c)

Fig. 5 Maximum and minimum nanohardness as a function of indentation depth for multilayers in inset. The other data points lie between the two curves but are left out for claririty of the plot: (a) TiN/AIN, (b) TiN/NbN and (c) TiN/TaN. 


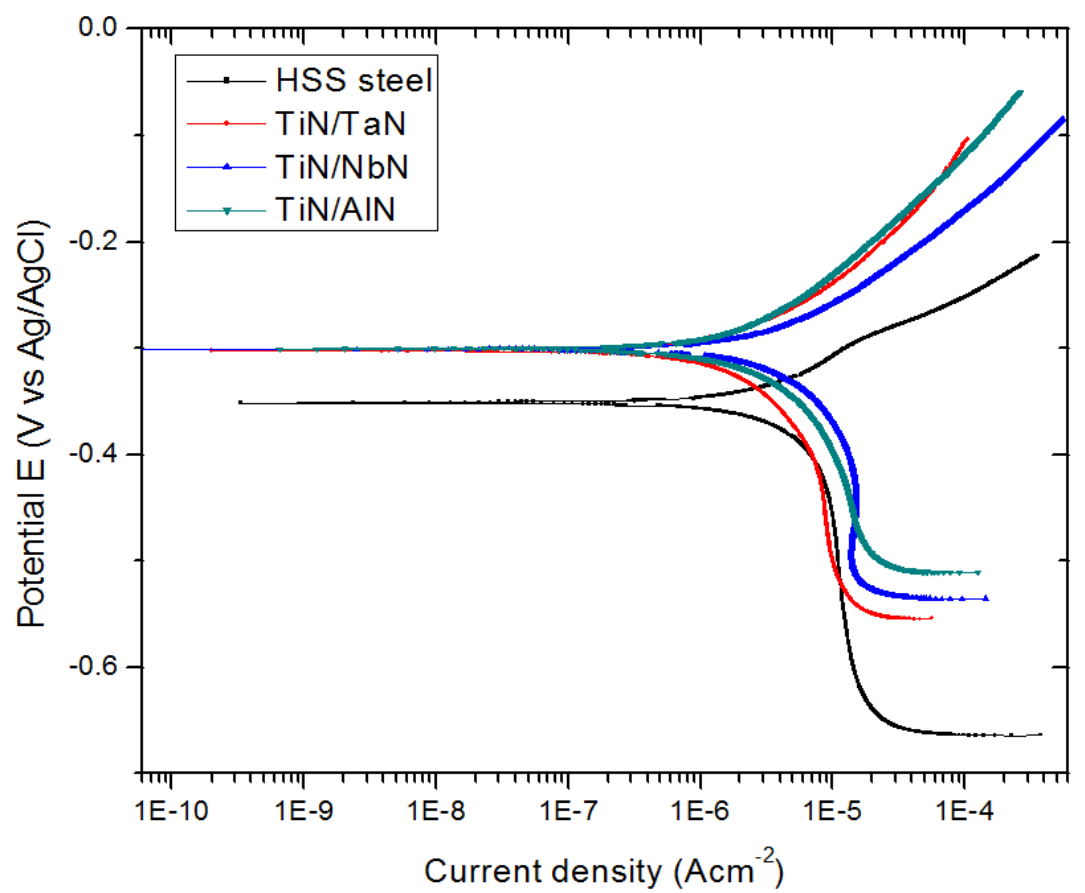

Fig. 6 Potentiodynamic polarization curves of uncoated HSS steel and multilayer coated and uncoated HSS steel in $0.10 \mathrm{M}$ $\mathrm{Na}_{2} \mathrm{SO}_{4}-0.01 \mathrm{M} \mathrm{NaCl}$ solution.

Table 5 The values of $E_{\text {corr }}$ and $i_{\text {corr }}$ uncoated steel and steel coated with each of the studied type of multilayers.

\begin{tabular}{lll}
\hline & $\mathrm{E}_{\text {corr }}(\mathrm{V})$ & $\mathrm{i}_{\text {corr }}\left(\mathrm{A} / \mathrm{cm}^{2}\right)$ \\
\hline HSS steel & -0.350 & $2.64 \times 10^{-6}$ \\
TiN/TaN & -0.301 & $1.7 \times 10^{-6}$ \\
TiN/NbN & -0.299 & $2.1 \times 10^{-6}$ \\
TiN-AlN & -0.299 & $2.3 \times 10^{-6}$ \\
\hline
\end{tabular}

the crystallographyc anisotropy.

\section{Electrochemical Corrosion}

Fig. 6 shows polarization curves for uncoated steel and steel coated with three types of multilayers. First observation is that $\mathrm{E}_{\text {corr }}$ for uncoated steel is $-0.35 \mathrm{~V}$ and for all the coated samples is the same, approximately, $-0.30 \mathrm{~V}$. The fact that $\mathrm{E}_{\text {corr }}$ is the same for all of our three types of multilayers can be explained because the top layer in all of them is a layer of approximately $80 \mathrm{~nm}$ of titanium nitride. The $\mathrm{E}_{\text {corr }}$ is a parameter to be considered mainly when in the surrounding of the piece of interest there is another material of very different $\mathrm{E}_{\text {corr }}$. In that case galvanic pairs may be stated and destroy the piece. On the other hand, the corrosion current density $\left(\mathrm{i}_{\text {corr }}\right)$ is a parameter related to microscopic currents occurring inside the piece of interest when in the medium of work or environment there exist some ionic solutions. The explanation for these microscopic currents is that due to defects of the material, there is accumulation of positive and negative charges in different points on the surface of the piece. In laboratory, $\mathrm{i}_{\text {corr }}$ is calculated from polarization curves according to the so called Tafel extrapolation method. Following this, we have found the values of $i_{\text {corr }}$ for uncoated steel and steel coated with each of the studied type of multilayers described in Table 5 . We can see that $i_{\text {corr }}$ for coated samples is only slightly lesser than for uncoated samples. The minimum $i_{\text {corr }}$ (maximum anticorrsosive protection) found is for TiN/TaN multilayers. In this case current corrosion is $64 \%$ current corrosion of uncoated steel.

\section{Discussion}

It is very well known that negative bias voltage between - 100 and - $200 \mathrm{~V}$ applied to high-speed-steel substrates during coating deposition by $\mathrm{dc}$ or $\mathrm{rf}$ magnetron sputtering of titanium nitride and other 
metal nitride increases noticeabely the hardness of the coatings [18-20]. This hardness increasing is generally associated to changes of texture respect to no-biased substrates and the creation of high density of deffects. On the other hand, superhard-multilayer coatings have been produced [21-24] by the intercalation of layers of two nitrides made also by sputtering with negative bias of the substrate. In the case of superhard-multilayer coatings, the hardness enhancement is usually explained by four mechanisms: (i) dislocation blocking $[8,25]$ due to the different shear moduli between two layers of different materials, (ii) the Hall-Petch effect [26] which explains the increase of hardness with decreasing grain size, (iii) alternating strain field due to the lattice mismatch [27] and (iv) enhancement of the elastic modulus [28].

In this work we have produced multilayers of TiN and $\mathrm{MeN}$ (where $\mathrm{Me}=\mathrm{Al}, \mathrm{Nb}$ and $\mathrm{Ta}$ ) under the same deposition conditions, including $-100 \mathrm{~V}$ bias substrate. We have shown microhardness data as measured by classical Vickers microdurometer, as well as, nanohardness data measured by nanoindentation with Berkovich tip. Vickers microhardness for load L $=$ $5 \mathrm{gf}$, according to column 4 of Table 2 , is in the range of $3,100 \mathrm{kgf} / \mathrm{mm}^{2}$ for TiN/NbN to $4,800 \mathrm{kgf} / \mathrm{mm}^{2}$ for TiN/TaN multilayers, however these values correspond to individual indentations. Considering only data on Table 4 for average nanohardness, we conclude that all of our multilayer systems show hardness enhancement respect to monolayers systems and there is no significative difference among them.

In order to explain the mechanisms for hardness enhancement in our coatings, we state the following: (1) From GDOES data, it is evident that we have a multilayer structure, i.e interfaces between materials with different elastic constants [29-32], then it is possible some contribution to hardness enhancement from dislocation blocking due to different shear moduli; (2) From our XRD data, it is evident that we have very small grain size, then it is possible in our coatings some contribution to hardness enhancement from Hall-Petch effect; (3) Lattice parameter of cubic tantalum nitride is very close to that of titanium nitride and some mismatch lattice could be expected; (4) The elastic modulus of our coatings are higher than respective monolayers so hardness enhancement could also be related to this fact.

It is not possible from our data to discriminate the specific contribution of each of the mentioned mechanisms, however, it is true that both, interfaces and grain boundaries, created by bias sputtering or mismatch, generate high defect density in the coatings. Deffects hinder the movement of dislocations, thus hardening the material. That could be a basic summary for explanation of hardness enhancement in our multilayer respect to monolayers coatings.

\section{Conclusions}

We have produced three types of multilayer coatings by dc magnetron sputtering with substrate biased at $-100 \mathrm{~V}$ and with the same sputter conditions, the layers type were: TiN/AIN, TiN/NbN and TiN/TaN. The thickness of titanium nitride layer was $80 \mathrm{~nm}$ titanium nitride and for the other nitride layers the thickness was between 13 and $16 \mathrm{~nm}$. The multilayers structure was verified by GDOES and nanocrystalline structure of each layer was verified by XRD. Microhardness and nanohardness were measured with classical Vickers microdurometer and by nanoindentation, respectively. Values of microhardness with load $\mathrm{L}=5$ gf result higher than nanohardness with indentation depth $100 \mathrm{~nm}$. In both cases (Vickers and nanoindentation) hardness enhancement with respect to the monolayers is evident. Average nanohardness taken over 16 indentations per sample results almost the same for the three types of multilayers, approximately $30 \mathrm{GPa}$. We conclude that the mechanisms for hardness enhancement are basically the same in the three types of layers: hindering of the movement of dislocations due to the high density of defects created by interfaces and grain boundaries. Electrochemical corrosion measurements 
were also carried out and the values of $\mathrm{E}_{\text {corr }}$ and $\mathrm{i}_{\text {corr }}$ are also almost the same for the three types of multilayers. So, the three types of multilayers based on titanium nitride and prepared under the same sputter conditions show similar values of nanohardness and the same electrochemical behavior independently of the partner layer: aluminium nitride, niobium nitride or tantalum nitride.

\section{Acknowledgments}

This paper is part of a research project financed by FINCyT, peruvian government, under contract $\mathrm{N}^{0}$ 139-FINCyT-IA-2013. We are also very thankful to Dr. Patrick Chapon from HORIBA Scientific company for GDOES measurements. Thanks are due also to colleagues Rafael Pujada from UNI, Lima, Perú, for fruitfull discussions and William Gacitua, from Bio-Bio University of Chile, for verification of nanoindentaion measurements.

\section{References}

[1] Zhang, S. et al. 2003. "Nanostructured Thin Films and Coatings: Mechanical Properties."

[2] Veprek, S. and Reiprich, S. 1995. "A Concept for the Design of Novel Superhard Coatings." Thin Solid Films 268: 64-71.

[3] Veprek, S. 1999. "The Search for Superhard Materials." J. Vac. Sci. Technol. A 17 (5): 2401-20.

[4] Veprek, S. and Jilek, M. 2002. "Superhard Composite Coating: from Basic Science toward Industrialization." Pure Appl Chem. 74 (3): 475-81.

[5] Ribeiro, E., Maiczyk, A., Carvalho, S., Rebouta, L., Fernandes, J. V. and Alves, E. 2002. "Effects of Ion Bombardment on Properties of DC Sputtered Superhard (Ti, Si, Al) N Nanocomposite Coatings." Surface and Coatings Technology 151-152: 515-20.

[6] Subramanian, B., Ananthakumar R. and Jayachandran, M. 2011. Cryst. Res. Technol. 46 (12): 1273-82.

[7] Barshilia, H. C. and Rajam, K. S. 2003. Bull. Mater Sci. 26 (2): 233-7.

[8] Auger, M. A., Sanchez, O., Ballesteros, C., Jergel, M., Aguilar, F. M. and Falcony, C. 2003. "TiN/AIN Bilayers and Multilayers Grown by Magnetron Co-Sputtering.” Thin Solid Films 433 (1): 211-6.

[9] Zeng, X. T., Zhang, S. and Hsieh, J. 1998. "Development of Graded Cr-Ti-N Coatings." Surface and Coatings Technology 102: 108-12.
[10] Oliver, W. C. and Pharr, G. M. 1992. "An Improved Technique for Determining Hardness and Elastic Modulus using Load and Displacement Sensing Indentation Experiments." J. Mater. Res. 7: 1564-70.

[11] Oswald, S. and Baunack, S. 2003. "Comparison of Depth Profiling Techniques Using Ion Sputtering from the Practical Point of View." Thin Solid films 425: 9-19.

[12] Nelis, T. and Payling, R. 2003. "Glow Discharge Optical Emission Spectrometry.” In Surface Analysis Methods in Materials Science of Springer Series in Surface Sciences, 23: 553-9.

[13] Scherrer, P. 1918. "Nachrichten von der Gesellschaft der Wissenschaften Goettingen." MathematischePhysikalische Klasse 2: 98-100.

[14] Guzman, M. S., Neubauer, G., Flinn, P. A. and Nix, W. D. 1993. "Materials Research Society." Mat. Res. Symp. Proc. 308.

[15] Patel, N. et al. 2014. "Secondary Hardness Enhancement in Large Period TiN/TaN Superlattices." Surrface \& Coatings Technology 254: 21-7.

[16] McCafferty, E. 2005. "Validation of Corrosion Rates Measured by the Tafel Extrapolation Method." Corrosion Science 47 (12): 3202-15.

[17] Tafel, J., Schmitz, K., Naremann, K. and Emmert, B. 1905. "Uber Die Polarization bei Kathodischer der Protonuebertragung." Z. Phys. Chem. 50: 641-752.

[18] Sproul, W. D., Rudnik, P. J. and Graham, M. E. 1989. "The Effects of N2 Partial Pressure, Deposition Rate and Substrate Bias Potential on the Hardness and Texture of Reactively TiN Coatings." Surface and Coating Technology 39/40: 355-63.

[19] Dudek, M., Zabeida, O., Klemberg, S. J. E. and Martinu, L. 2009. "Effect of Substrate Bias on the Micro- Structure and Properties of Nanocom-Posite Titanium Nitride Based Films." Journal of Achievements in Materials and Manufacturing Engineering 37: 416-21.

[20] Ait-Djafter, A. Z., Saolua, N., Aknouche, H., Guedouar, B. and Madaoui, N. 2015. "Deposition and Characterization of Titanium Aluminum Nitride Coatings Prepared by RF Magnetron Sputtering." Applied Surface Science 350: 6-9.

[21] Ou, Y. X., Lin, J., Tong, S., Che, H. L., Sproul, W. D. and Lei, M. K. 2015. "Wear and Corrosion Resistance of CrN/TiN Superlattice Coatings Deposited by a Combined Deep Oscillation Magnetron Sputtering and Pulsed dc Magnetron Sputtering." Applied Surface Science 351: 332-43.

[22] Zhang, S. H., Byon, E., Li, M. X., He, Y. Z., Cai, F. and Wang, L. 2011. "Realization of Superhard Nanocomposites with Sufficient Toughness: Superlattice Nanocrystal-TiN/Amorphous-(W, Ti) $\mathrm{C}_{0.83}$ Films." Thin Solid Films 519: 1901-6. 
[23] Zhang, S. H., Li, J. L., Chen, Z. and Li, M. X. 2012. "Realization of Superhard Chromium Nitride-Based Films: A Superlattice Nanocrystalline - $\mathrm{Cr}_{2} \mathrm{~N} /$ Amorphous - WC Film.” Thin Solid Films 520: 4984-9.

[24] Lin, J. L., Moore, J. J., Mishra, B., Pinkas, M., Zhang, X. H. and Sproul, W. D. 2009. "CrN/AlN Superlattice Coatings Synthesized by Pulsed Closed Field Unbalanced magnetron Sputtering with Different $\mathrm{CrN}$ Layer Thicknesses." Thin Solid Films 517: 5798-804.

[25] Chu, X. and Barnettt, S. A. 1995. "Model of Superlattice Yield Stress and Hardness Enhancement." J. Appl. Phys. 77: 4403-41.

[26] Anderson, P. M. and Li, C. 1995. Nanostruct. Mater. 5:
349.

[27] Kato, M., Mori, T. and Schwartz,. L. H. 1980. "Hardening by Spinodal Modulated Structure.” Acta Metall. 28: 285-90.

[28] Cammaratta, R. C., Schlesinger, T. E., Kim, C., Qadri, S. B. and Edelstein. A. S. 1990. Appl. Phys. Lett. 56: 1862-4.

[29] Chen, D. et al. 2009. Acta Metall. Sin. (Engl. Lett.) 22 (2): 146-52.

[30] Gerlich, D., Dole, S. L. and Slack, G. A. 1986. "Elastic Properties of Aluminum Nitride." J. Phys Chem Solids 47 (5): 437-41.

[31] Chang, J. et al. 2012. J. Appl Phys. 112 (8): 0835519.

[32] Dahua, R. et al. 2014. "Advanced Materials." Journal of Wuhan University of Technology Mater. Sci. 29: 49-57. 